\title{
Clinical significance of detecting circulating tumor cells in patients with esophageal squamous cell carcinoma by EpCAM-independent enrichment and immunostaining-fluorescence in situ hybridization
}

\author{
YAOWEN ZHANG ${ }^{1,2^{*}},{\text { JIAN } \mathrm{LI}^{3 *}, \mathrm{LU}^{*} \mathrm{WANG}^{4,5}, \text { PENG MENG }^{4,5} \text {, JIANGMAN ZHAO }}^{4,5}$, PENG HAN $^{6}$, JIN XIA $^{7}$, \\ JIANGONG XU ${ }^{3}$, LIDONG WANG ${ }^{8}$, FANGFANG SHEN ${ }^{9}$, ANPING ZHENG ${ }^{2}$, FUYOU ZHOU $^{3}$ and RUITAI FAN ${ }^{1}$ \\ ${ }^{1}$ The First Department of Radiation Oncology, The First Affiliated Hospital of Zhengzhou University, \\ Zhengzhou, Henan 450003; ${ }^{2}$ The First Department of Radiation Oncology, ${ }^{3}$ Department of Thoracic Surgery, \\ Anyang Tumor Hospital, Anyang, Henan 455000; ${ }^{4}$ Department of Medicine, Shanghai Zhangjiang Institute of \\ Medical Innovation; ${ }^{5}$ Biotecan Pharmaceuticals Co., Ltd., Shanghai 201204; ${ }^{6}$ Department of Pathology, \\ ${ }^{7}$ The Fifth Department of Oncology, Anyang Tumor Hospital, Anyang, Henan 455000; ${ }^{8}$ Henan Key Laboratory for \\ Cancer Research, The First Affiliated Hospital of Zhengzhou University, Zhengzhou, Henan 450003; \\ ${ }^{9}$ The Key Laboratory for Tumor Translational Medicine, The Third Affiliated Hospital \\ of Xinxiang Medical University, Xinxiang, Henan 453003, P.R. China
}

Received December 13, 2018; Accepted May 14, 2019

DOI: $10.3892 / \mathrm{mmr} .2019 .10420$

\begin{abstract}
Circulating tumor cells (CTCs) are tumor cells present in the bloodstream, which originate from tumor sites, and are ultimately responsible for metastasis or relapse in several types of cancer. However, to the best of our knowledge, only a few studies have investigated these extremely rare cells in esophageal squamous cell carcinoma (ESCC). In the present study, 63 patients with ESCC and 50 healthy donors were recruited, and the potential clinical significance of CTCs was assessed using subtraction enrichment and immunostaining-fluorescence in situ hybridization. Blood samples were collected at the following times: At first diagnosis, following neoadjuvant chemoradiotherapy, $24 \mathrm{~h}$ and 13 days post-surgery, and every 3 months during follow-up. Cytokeratin (CK)-positive and clustered CTCs only accounted
\end{abstract}

Correspondence to: Dr Fuyou Zhou, Department of Thoracic Surgery, Anyang Tumor Hospital, 1 North Huanbin Road, Anyang, Henan 455000, P.R. China

E-mail: ayzhoufuyou@163.com

Dr Ruitai Fan, The First Department of Radiation Oncology, The First Affiliated Hospital of Zhengzhou University, 1 East Jianshe Road, Zhengzhou, Henan 450003, P.R. China

E-mail: fanruitai@126.com

${ }^{*}$ Contributed equally

Key words: circulating tumor cells, esophageal squamous cell carcinoma, relapse for $1 \%$ of total CTCs detected, whereas most CTCs were CK-negative aneuploid cells. Patients with ESCC $(n=63)$ had higher CTC counts compared with healthy donors (control group; $n=50$ ) (area under curve $=0.807$, median CTC count, 2 vs. 0 ). However, there was no statistical association between CTC counts and sex, age, pathological stage, tumor location, tumor depth or lymph node involvement $(\mathrm{P}>0.05)$. The association of tumor development with CTC status and other circulating biomarkers was monitored in patients for a further 2 years. The results revealed that a change in CTC counts between first diagnosis and 13 days post-surgery $(\triangle \mathrm{CTC})$ of $\geq 2 / 7.5 \mathrm{ml}$ peripheral blood could be applied for predicting progression-free survival (hazard ratio, 3.922; 95\% confidence interval, 0.907-16.951; $\mathrm{P}<0.05)$ in patients with ESCC. In conclusion, $\triangle \mathrm{CTC}$ evaluation may be a promising indicator for predicting tumor prognosis and the clinical efficacy of treatment in patients with ESCC.

\section{Introduction}

Esophageal carcinoma is a malignant tumor originating from esophageal epithelium, which is characterized by strong invasiveness and a high mortality rate (1). The incidence of esophageal cancer ranks eighth among all types of cancer worldwide (2). A total of $80-85 \%$ of esophageal cancer cases occur in developing countries, including China (3). Esophageal squamous cell carcinoma (ESCC) is the major histopathological subtype of esophageal cancer $(4,5)$, and accounts for the majority of esophageal cancer cases in China (6,7). Despite great advances in medical and surgical treatments, the prognosis of patients remains poor, with an overall 5 -year survival rate of $15-25 \%(8,9)$. 
Currently, the clinical diagnosis of ESCC is mainly based on serum protein tumor markers, endoscopy and histopathological methods (10). The application of serological tumor markers in clinical diagnosis, including SCC antigen and carcinoembryonic antigen (CEA), is limited due to low specificity and accuracy (11). As well as reduced sensitivity for small-diameter tumor lesions, endoscopy causes patients a certain degree of pain $(10,12,13)$. In addition, although histopathology remains the gold standard for tumor diagnosis, it cannot be used to dynamically monitor treatment response, since patients who have undergone surgery are unable to provide diseased tissue during this process (14). Therefore, an effective adjuvant diagnostic method is required to detect early esophageal cancer, monitor treatment response and predict prognosis.

Circulating tumor cells (CTCs) are tumor cells that have moved from the primary tumor site into the circulatory system, which usually have a strong invasive and metastatic capacity (15-17). The enumeration of CTCs and their phenotypes may provide useful information for the diagnosis, treatment and prognosis of malignant tumors $(15,18-20)$. The CellSearch ${ }^{\circledast}$ system was previously the only instrument approved by the US Food and Drug Administration for evaluating CTCs in patients with breast cancer (21), and is based on epithelial cell adhesion molecule (EpCAM) and cytokeratin (CK) expression in the tumor cell itself (22). However, EpCAM is highly heterogeneous and dynamically expressed in various epithelial tumor cells, and epithelial-mesenchymal transition may reduce EpCAM and CK expression, resulting in failure of CTC detection (23). Furthermore, the system cannot detect tumor cells that are hyperdiploid and tumor marker-negative.

To promote further research and clinical applications of CTC detection, other platforms, including immunostaining-fluorescence in situ hybridization (iFISH) have been introduced. Compared with CellSearch ${ }^{\circledast}$, iFISH can effectively detect chromosome ploidy, and tumor markers in the cytoplasm or on the cell surface $(24,25)$. Furthermore, numerous studies have indicated that iFISH has a high CTC detection rate $(15,19,24,25)$. Therefore, iFISH may be considered a more promising method for detecting CTCs in patients with ESCC.

In the present study, subtraction enrichment (SE) and iFISH were used to detect CTCs from the peripheral blood (PB) of patients with ESCC. SE was used to acquire the CTCs, whereas iFISH was performed to identify them. The association between CTC status and the clinicopathological features or prognosis of patients with ESCC was subsequently evaluated.

\section{Materials and methods}

Patients and sample collection. The present study was performed at Anyang Cancer Hospital. A total of 63 patients with confirmed esophageal cancer (stage 0 and I, 11; stage II, 27; stage III, 25) and 50 healthy donors (age, 40-72; male:female, $35: 15)$ were enrolled in the present study between November 2015 and July 2017. A blood (7.5 ml) CTC test was performed on patients with ESCC at the time of first diagnosis, after neoadjuvant chemoradiotherapy (NCRT), $24 \mathrm{~h}$ and 13 days post-surgery, and every 3 months during the follow-up period.

This study was approved by the Ethics Committee of Anyang Cancer Hospital. Written informed consent was obtained from all subjects. Clinical data were collected with regards to sex, age, primary tumor site, tumor size, lymph node metastasis (LNM), histological type, CEA and SCC antigen levels, and progressive disease. Tumor-node-metastasis (TNM) staging was performed according to the American Joint Committee on Cancer 2010 staging system (26). Patients were subjected to endoscopic ultrasound along-with chest and abdominal enhanced computerized tomography (CT) scans to carry out pre-operative assessments of the clinical stage of ESCC and the status of distant metastasis. To evaluate tumor responses, an endoscopic biopsy and chest CT were carried out one month following the completion of therapy. A follow-up CT and an esophagography were carried out every 3 months for the first 2 years, and every 6 months thereafter. Serial PB samples $(7.5 \mathrm{ml})$ were collected from each subject. The samples were tested within $48 \mathrm{~h}$ of collection.

SE analysis. CTC enrichment was performed using the Human Circulating Rare Cell Subtraction Enrichment kit (Cytelligen, Inc.), according to the manufacturer's protocol. PB samples $(7.5 \mathrm{ml})$ were centrifuged at $800 \mathrm{xg}$ for $8 \mathrm{~min}$ at room temperature, and the supernatant above the red blood cell layer was discarded. hCTC Separation Matrix ( $3 \mathrm{ml}$ ) was mixed with the remaining components, and the mixture was centrifuged at $450 \mathrm{xg}$ for $8 \mathrm{~min}$ at room temperature. After centrifugation, the white buffy coat was collected and incubated with $150 \mu \mathrm{l}$ anti-CD45 monoclonal antibody-conjugated immunomagnetic particles at room temperature for $20 \mathrm{~min}$ with gentle shaking, which then underwent magnetic separation to remove leukocytes. The solution without beads was transferred to a clean centrifuge tube and centrifuged at $450 \mathrm{x}$ g for $8 \mathrm{~min}$ at room temperature, and then washed twice. The resulting cell pellet was mixed with cell fixative and applied to coated CTC slides. After drying at $32^{\circ} \mathrm{C}$ for $4 \mathrm{~h}$, the slides were identified using iFISH.

Immunofluorescence staining of CTCs. CTC identification was performed according to the instructions of the Cytelligen CTC Enrichment kit (cat. no. SEH-003; Cytelligen, Inc.). The slides were immersed in $2 \mathrm{X}$ saline-sodium citrate buffer for $10 \mathrm{~min}$ and dehydrated in ethanol for $2 \mathrm{~min}$. Centromere Probe 8 (CEP8) Spectrum Orange (Abbott Laboratories) was added to the slides, denatured at $76^{\circ} \mathrm{C}$ for $5 \mathrm{~min}$ and hybridized for $90 \mathrm{~min}$ at $37^{\circ} \mathrm{C}$. Subsequently, the slides were incubated with Antibody Preparation Solution-1, Alexa Fluor $^{\circledR}$ 594-conjugated anti-CD45 immunoglobulin G (IgG) and Alexa Fluor ${ }^{\circledR}$ 488-conjugated anti-CK18 IgG (1:200). After incubation at room temperature for $2 \mathrm{~h}$ in the dark, the slides were washed and mounted with DAPI (Vector Laboratories, Inc.) containing mounting medium. Finally, the CTCs were detected under a fluorescence microscope with a x100 oil immersion objective (Olympus Corporation).

Statistical analysis. Data were analyzed using SPSS 23.0 (IBM Corp.). Differences in CTC numbers between the healthy controls and patients were compared using nonparametric Mann-Whitney U test. One-way ANOVA and Bonferroni multiple comparisons test were performed to analyze differences in CTC counts among tumor stages. Graphical plots were generated using GraphPad Prism 6.0 
(GraphPad Software, Inc.) and OriginPro8.1 (OriginLab Corporation). Receiver operating characteristic (ROC) curves were plotted to analyze the sensitivity and specificity of CTCs between patients with ESCC and healthy controls. Spearman's correlation analysis was applied to analyze the correlation between CTC and serological tumor markers. Kaplan-Meier survival curves and log-rank test were used to compare the differences in progression-free survival (PFS) rate between two groups. $\chi^{2}$ was used to determine the relationship between CTCs and clinicopathological characteristics of esophageal SCC. Univariate and multivariate Cox proportional hazards regression analyses were carried out to identify independent risk factors for clinical outcomes. A two-sided $\mathrm{P}<0.05$ was considered to indicate a statistically significant difference.

\section{Results}

Identification of CTCs from patients with ESCC. The cells obtained through SE were identified based on chromosome ploidy, existence of a nucleus, a hematopoietic white blood cell (WBC) marker and an epithelial marker, using CEP8, DAPI, CD45 and CK, respectively. CTCs were characterized as hyperdiploid cells without detectable CD45 expression. In particular, CTCs in the present study were defined as follows: $\mathrm{DAPI}^{+} / \mathrm{CD} 45 / \mathrm{CK}^{+} / \mathrm{CEP} 8>2$ spots, $\mathrm{DAPI}^{+} / \mathrm{CD} 45 / \mathrm{CK}^{+} / \mathrm{CEP} 8=2$ spots, or $\mathrm{DAPI}^{+} / \mathrm{CD} 45 / \mathrm{CK}^{-} / \mathrm{CEP} 8>2$ spots. $\mathrm{WBCs}$ were defined as $\mathrm{CK}^{-} / \mathrm{CD}^{+} 5^{+} / \mathrm{DAPI}^{+} / \mathrm{CEP} 8=2$ spots, and indeterminate cells were defined as $\mathrm{CK}^{-} / \mathrm{CD}^{\circ} / 5^{\circ} \mathrm{DAPI}^{+} / \mathrm{CEP} 8=2$ spots (Fig. 1A-E). Only FISH signals with homogeneous light and size were counted as a spot as previously described $(27,28)$.

According to the current criteria, a total of 292 CTCs were detected from the 63 patients with ESCC. $\mathrm{CK}^{+} \mathrm{CTC}$ s have been reported to account for a relatively small proportion of CTCs in breast and colorectal cancer $(28,29)$. As expected, only two CTCs were found to be $\mathrm{CK}^{+}$, occurring in two patients (Fig. 1E). The majority of CTCs were $\mathrm{CK}^{-} / \mathrm{CD}^{2} / / \mathrm{DAPI}^{+} / \mathrm{CEP} 8$ $>2$, which accounted for $99.3 \%$ (290/292) of all CTCs in the first diagnosis group. Among them, 50 CTCs ( $\mathrm{n}=22$ patients) were triploid (Fig. 1A), 50 CTCs ( $\mathrm{n}=25$ patients) were tetraploid (Fig. 1B), 37 CTCs ( $\mathrm{n}=14$ patients) were pentaploid and 153 CTCs $(n=37$ patients) were multiploid ( $>5$ copies of chromosome 8; Fig. 1C and F). In addition, two CTC clusters were identified in two patients at first diagnosis (Fig. 1D). CTC clusters are recognized as oligoclonal precursors of breast cancer metastasis (30); however, to the best of our knowledge, they have not yet been studied in ESCC. Although no direct association was observed in the present study, due to limited sample size, further studies with a larger sample size are required, in order to provide further insight into the effects of CTCs on ESCC prognosis.

CTCs in patients and controls. The distribution of CTCs in patients with ESCC and healthy controls was subsequently examined. There was a significant difference between the CTC status of patients and that of controls ( $\mathrm{P}=0.0003$; Fig. 2A). To evaluate the validity of CTC detection in discriminating between patients and healthy controls, ROC curves were used. The cut-off value of $1 \mathrm{CTC} / 7.5 \mathrm{ml}$ yielded $74.6 \%$ sensitivity and $74.0 \%$ specificity. The area under the ROC curve (AUC) was 0.807 [95\% confidence interval (CI), 0.727-0.887], which is similar to that of CEA and SCC antigen (Fig. 2B). Taking into consideration the actual cut-off value of CEA $(5 \mu \mathrm{g} / \mathrm{l})$ and SCC $(2.5 \mu \mathrm{g} / \mathrm{l})$ in terms of clinical application, the sensitivity of CTCs with a cut-off value of $1 \mathrm{CTC} / 7.5 \mathrm{ml}$ was much higher (SCC, 22.2\%; CEA, 4.8\%). In addition, using a logistic regression model, it was predicted that the optimal AUC could be further improved to 0.941 by combining all three biomarkers; suggesting that CTC may serve as a better biomarker when combined with traditional serum biomarkers. The association between CTC counts and several ESCC clinicopathological variables was also compared. The median CTC count per $7.5 \mathrm{ml}$ PB in stage 0 -I, stage II and stage III disease was six (range, 0-12), five (range, 0-10), and nine (range, 0-18), respectively; however, the difference was not significant ( $P>0.05$; Fig. 2C). The median CTC count per $7.5 \mathrm{ml}$ $\mathrm{PB}$ in patients with positive and negative LNM was nine (range, $0-18$ ) and six (range, 0-12), respectively, and the difference was not significant ( $\mathrm{P}=0.693$; Fig. 2D). Similarly, CTC status was not significantly associated with sex, age, pathological stage, tumor location, tumor depth or lymph node involvement $(\mathrm{P}>0.05$; Table I). In addition, the correlation between CTC counts and the commonly used protein tumor markers, CEA and SSC, was investigated in patients, and it was suggested to be a relatively independent factor (Fig. 3).

\section{CTCs and other circulating biomarkers for tumor monitoring.} The presence of CTCs may provide important information for evaluating the clinical response to treatment, including surgery, chemotherapy and NCRT. To evaluate the differences in CTC counts, the CTCs of patients with ESCC were detected at numerous time points pre- or post-surgery (Fig. S1). The CTC counts of patients receiving NCRT $(n=12)$ were decreased compared with at first diagnosis $(n=63)$, suggesting that NCRT may exert effects on tumor regression. CTCs were detected in 51 out of 63 patients $24 \mathrm{~h}$ post-surgery and the counts were even higher compared with at first diagnosis. Further analysis of CTCs from 43 patients at the two time points is presented in Fig. S2. CTCs were detected in 43 patients prior to surgery and $24 \mathrm{~h}$ post-surgery. In accordance with the aforementioned results, CTC counts increased following surgery in $>50 \%$ of patients (22/43), whereas only 17 patients exhibited reduced CTC counts, and four had consistent CTC counts. No significant association with disease progression was observed. This finding may be explained by dislodged cells from surgery, which may affect the accuracy of the prognostic prediction. In addition, CTC counts gradually stabilized on day 13, and remained relatively stable between 3 and 12 months post-surgery.

To identify patients likely to suffer from recurrence following surgical intervention, serial plasma samples from six of the 63 patients with relapsed ESCC were collected and CTC counts were periodically detected across $\geq 2$ time points during 18 months of follow-up. The alterations in CTCs and other biomarkers, including SCC antigen and CEA, were compared alongside the results of clinical CT scans, which is the gold standard for measurement of disease status, as defined by the Response Evaluation Criteria in Solid Tumors 1.0 (RECIST 1.0) (31). CTCs were detected and exhibited serial alterations, alongside fluctuations associated with treatment response observed by CT. During follow-up, recurrence was detected by CT in several patients with increased CTC 
A

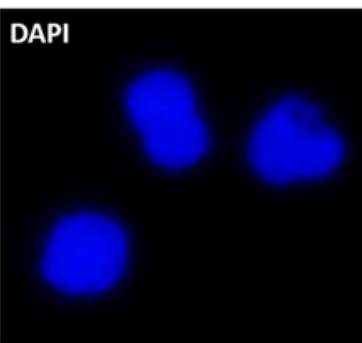

B

$\mathrm{C}$

$\mathrm{D}$

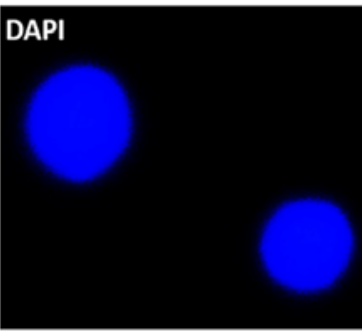

\section{DAPI}
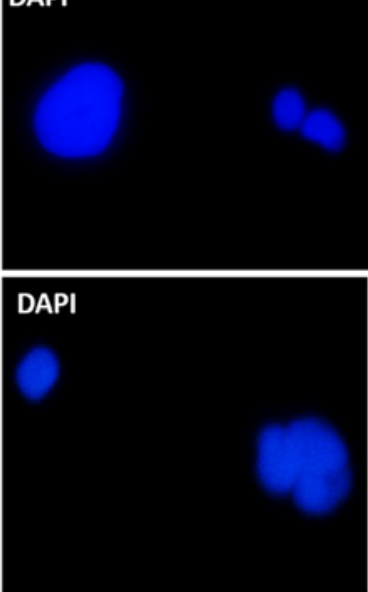

E

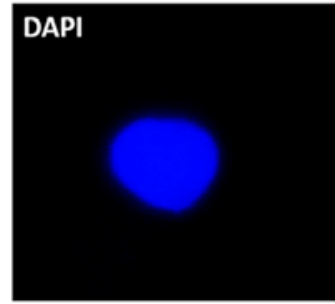

CEP8

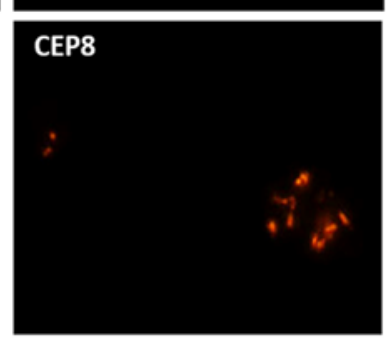

CEP8

$\bullet$

$\therefore$

\section{CEP8}

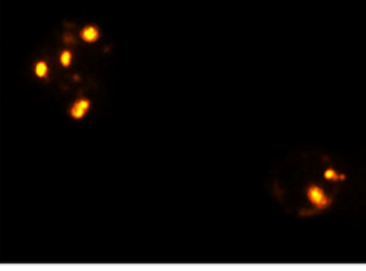

$\therefore$

(2)
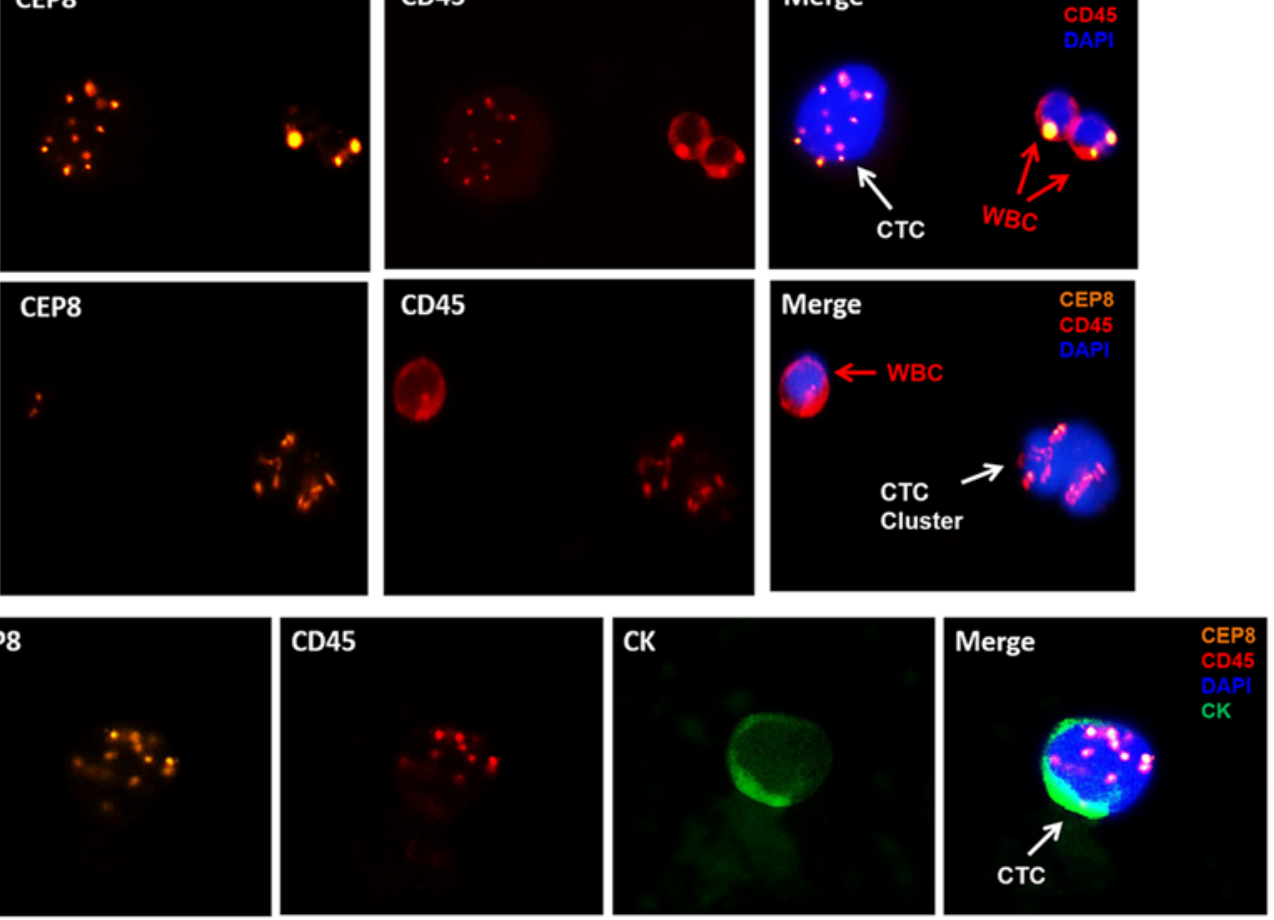

F $\quad 1 \%$

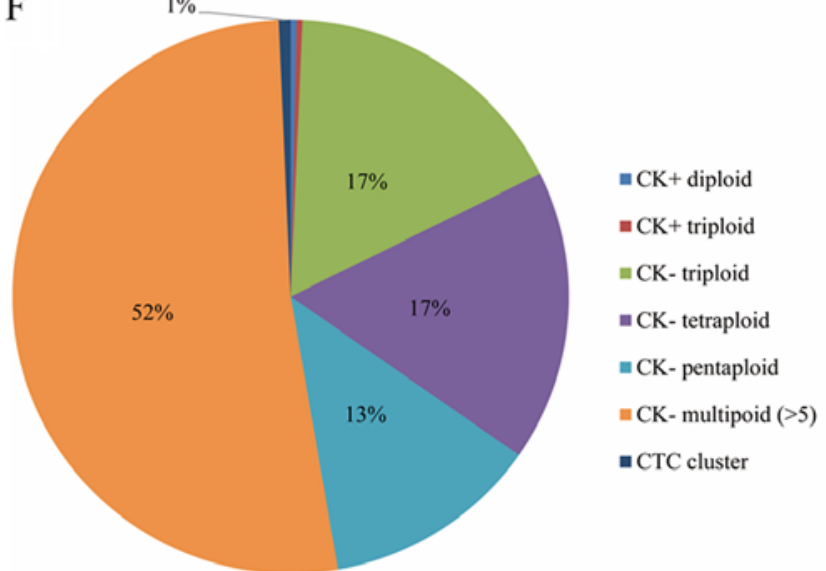

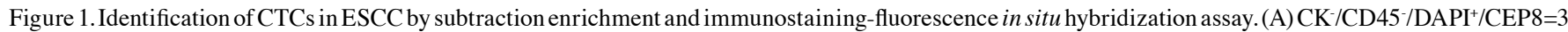
spots (white arrow), (B) $\mathrm{CK}^{-} / \mathrm{CD} 45^{-} / \mathrm{DAPI}^{+} / \mathrm{CEP} 8=4$ spots (white arrow), (C) $\mathrm{CK}^{-} / \mathrm{CD}^{-} 5^{-} / \mathrm{DAPI} / \mathrm{CEP} 8>5$ spots (white arrow), (D) $\mathrm{CTC}^{+}$cluster (white arrow), defined as $>2$ CTCs adhered together. (E) $\mathrm{CK}^{+} / \mathrm{CD} 45 / \mathrm{DAPI} / \mathrm{CEP} 8>2$ spots (white arrow). CK, green staining; CEP8, orange staining; DAPI, blue staining; CD45, red staining. Magnification, x400. (F) Distribution of CK expression and ploidy in the 292 CTCs from 63 patients with ESCC at first diagnosis. CEP8, Centromere Probe 8; CTC, circulating tumor cells; CK, cytokeratin; ESCC, esophageal squamous cell carcinoma; WBC, white blood cell. 
A

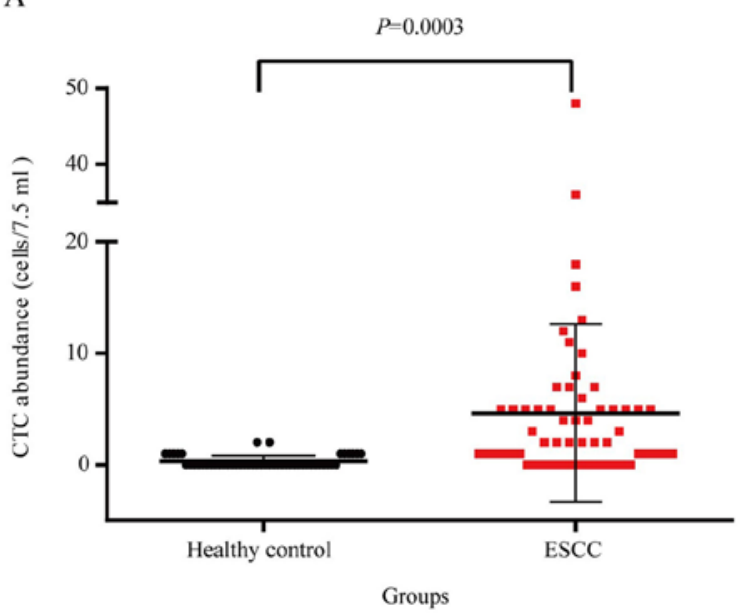

$\mathrm{C}$

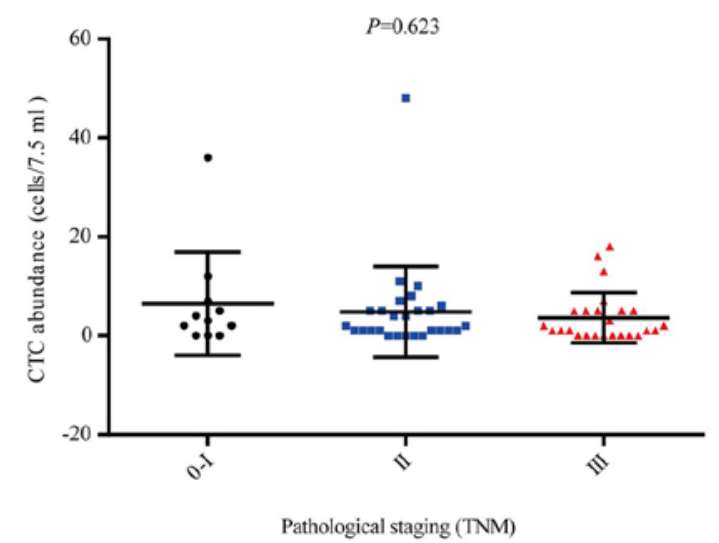

B

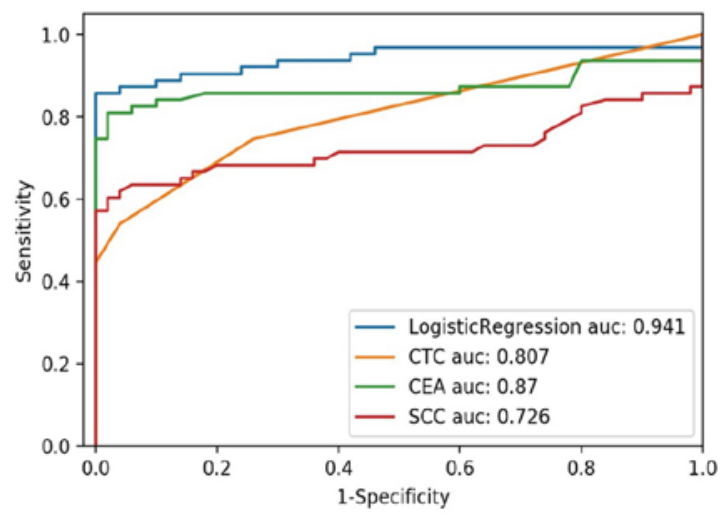

D

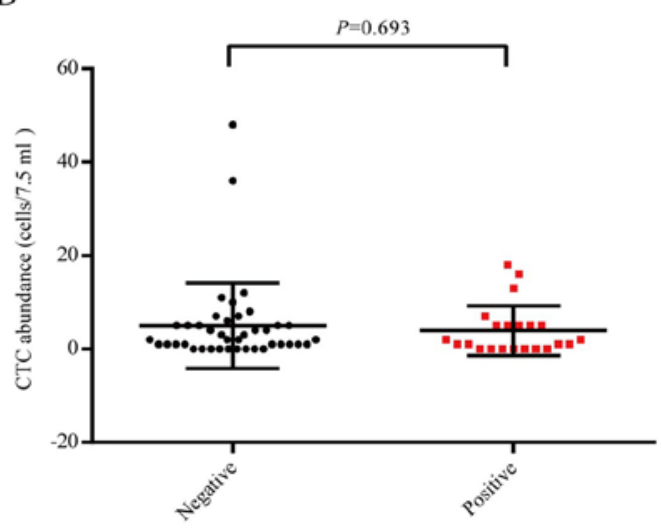

Lymph node metastasis

Figure 2. CTC counts in patients with ESCC and healthy controls. (A) Distribution of CTCs in diagnosed patients with ESCC and healthy controls. The existence of CTCs in 63 patients with ESCC and 50 healthy controls was examined. (B) Receiver operating characteristic curves for CTC counts, CEA, SCC antigen and the three combined markers (logistic regression model), were used to discriminate between patients with ESCC and healthy controls. The cut-off value was defined as $1 \mathrm{CTC} / 7.5 \mathrm{ml}$. (C) Distribution of CTCs in patients with ESCC according to pathological staging (TNM). (D) Distribution of CTCs in patients with ESCC according to lymph node metastasis. AUC, area under curve; CEA, carcinoembryonic antigen; CTCs, circulating tumor cells; ESCC, esophageal SCC; SCC, squamous cell carcinoma; TNM, Tumor-Node-Metastasis.

counts as early as 13 days post-surgery (Fig. 4A-C, E and F), suggesting that CTC counts on day 13 post-surgery may serve as a better indicator for prognosis. In the case of Patient 12, although the level of CTC on day 13 post-surgery was missing, the CTC counts appeared higher at day 1-180 and then gradually reduced. In addition, a marked increase in CTCs was observed at day 360 compared with day 270 post-surgery in Patient 12 (Fig. 4D), who had progressive disease according to a CT scan performed 90 days later. Reduced CEA/SCC antigen levels and a smaller dynamic range neither predicted nor reflected the presence of progressive disease in these patients. These data suggested that alterations in CTC counts may be a highly specific approach for the early detection of residual or recurrent disease following surgical resection.

Survival analysis. The median follow-up time of the 63 patients with ESCC was 21 months (range, 0-32 months). A total of 15 patients with ESCC had died or exhibited disease progression at the end point of PFS analysis (follow up time: median, 10 months; range 0-18 months). The median follow-up time of the remaining ESCC patients without progression was 24 months (range, 12-32 months). PFS survival curves were plotted to evaluate the risk of CTC counts at first diagnosis, and the alterations in CTC counts 13 days post-surgery $\left(\mathrm{CTC}_{13}\right)$ compared with at first diagnosis $\left(\mathrm{CTC}_{\mathrm{FD}}\right)\left(\Delta \mathrm{CTC}=\mathrm{CTC}_{13}-\mathrm{CTC}_{\mathrm{FD}}\right)$, using Kaplan-Meier analysis and the log-rank test. The results indicated that $\triangle \mathrm{CTC} \geq 2 / 7.5 \mathrm{ml}$ was an independent risk factor for reduced PFS in patients with ESCC ( $<<0.05$; Fig. 5).

CTC enumeration and other clinical factors, including sex, age, TNM staging, LNM and CEA levels were subjected to univariate Cox proportional hazards regression analysis to evaluate the potential association with PFS. Only factors with $\mathrm{P}<0.1$ were included in the multivariate Cox regression analysis (Table II). As a result, $\triangle \mathrm{CTC} \geq 2$, TNM staging, LNM, smoking and drinking qualified for further analysis. Consistent with the Kaplan-Meier analysis, $\Delta$ CTC $\geq 2 / 7.5 \mathrm{ml}$ remained a strong predictor of poor prognosis [hazard ratio, (HR), 3.922; 95\% CI: 0.907-16.951; $\mathrm{P}=0.047]$ in the multivariate Cox proportional hazards regression analysis.

\section{Discussion}

CTCs are tumor cells that enter the bloodstream from primary or metastatic lesions, which form at the early stage of cancer 
Table I. Relationship between CTCs and clinicopathological characteristics of esophageal SCC.

\begin{tabular}{|c|c|c|c|c|}
\hline Characteristics & $\mathrm{N}$ & $\mathrm{CTC}>0(\%)$ & $\mathrm{CTC}=0(\%)$ & P-value ${ }^{a}$ \\
\hline \multicolumn{5}{|l|}{ Sex } \\
\hline Male & 46 & 32 & 14 & 0.131 \\
\hline Female & 17 & 15 & 2 & \\
\hline \multicolumn{5}{|l|}{ Age (years) } \\
\hline$\geq 65$ & 26 & 21 & 5 & 0.346 \\
\hline$<65$ & 37 & 26 & 11 & \\
\hline \multicolumn{5}{|l|}{ Smoking history } \\
\hline Yes & 34 & 25 & 9 & 0.832 \\
\hline No & 29 & 22 & 7 & \\
\hline \multicolumn{5}{|l|}{ Pathologic stage } \\
\hline $0-\mathrm{I}$ & 11 & 8 & 3 & 0.53 \\
\hline II & 27 & 22 & 5 & \\
\hline III & 25 & 17 & 8 & \\
\hline \multicolumn{5}{|l|}{ Tumor location } \\
\hline Upper & 9 & 8 & 1 & 0.257 \\
\hline Middle & 45 & 34 & 11 & \\
\hline Lower & 9 & 5 & 4 & \\
\hline \multicolumn{5}{|l|}{ Tumor depth } \\
\hline Tis+T1 & 10 & 8 & 2 & 0.669 \\
\hline $\mathrm{T} 2+\mathrm{T} 3+\mathrm{T} 4$ & 53 & 39 & 14 & \\
\hline \multicolumn{5}{|l|}{ Lymph nodes } \\
\hline Negative & 41 & 32 & 9 & 0.391 \\
\hline Positive & 22 & 15 & 7 & \\
\hline \multicolumn{5}{|c|}{ Serum CEA (ng/ml) } \\
\hline$\geq 5.09$ & 3 & 1 & 2 & 0.114 \\
\hline$<5.09$ & 56 & 42 & 14 & \\
\hline Missing & 4 & 4 & 0 & \\
\hline \multicolumn{5}{|c|}{ Serum SCC antigen (ng/ml) } \\
\hline$\geq 2.5$ & 13 & 11 & 2 & 0.468 \\
\hline$<2.5$ & 44 & 33 & 11 & \\
\hline Missing & 6 & 3 & 3 & \\
\hline
\end{tabular}

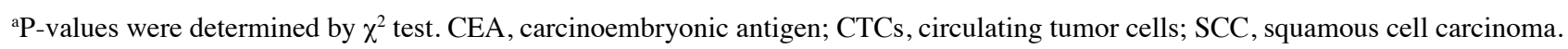

occurrence and metastasis (32). CTCs in the peripheral circulation exist independently or as cell clumps (30). Compared with tissue biopsy, CTC detection, the gold standard for cancer detection, is relatively noninvasive, easy to perform and can be performed repeatedly (33). CTC detection has been evaluated as a novel prognostic method and as a tumor marker for patients with various types of solid tumors, including esophageal cancer (25).

CTC detection involves the enrichment and discrimination of CTCs (34). Conventional enrichment, depending on surface antigens (35) and tumor cell size (36), can easily result in the loss of a number of CTCs. In the present study, SE was used to obtain CTCs. In this method, interfering components can be removed effectively, including WBCs, red blood cells and plasma proteins, through centrifugation and immunomagnetic separation (28). All types of CTCs and circulating tumor microemboli can be obtained, with the extraction having a less detrimental effect $(28,37)$. Therefore, SE is considered an ideal method for CTC enrichment and provides a good basis for further CTC analysis; Qiao et al (20) also applied this method to evaluate the prognostic value of CTCs in the PB of patients with ESCC. The SE-enriched CTCs were then stained with anti-CK8/18/19 and anti-CD45 antibodies. Compared with previous literature (20), the present study used a different staining technique for the enriched CTCs.

In the present study,iFISHwas used toidentify CTCs.Cells that were $\mathrm{CK}^{+} / \mathrm{DAPI}^{+} / \mathrm{CD} 45^{-} / \mathrm{CEP} 8=2$ spots, $\mathrm{CK}^{+} / \mathrm{DAPI}^{+} / \mathrm{CD}^{\circ} / \mathrm{CEP} 8$ $>2$ spots, and $\mathrm{CK}^{-} / \mathrm{CD} 45 / \mathrm{DAPI}^{+} / \mathrm{CEP} 8>2$ spots were considered CTCs, since they were hyperdiploid and/or tumor markerpositive. Cells that were $\mathrm{CK}^{-} / \mathrm{DAPI}^{+} / \mathrm{CD} 45^{+} / \mathrm{CEP} 8=2$ spots were considered to be WBCs $(38,39)$, whereas cells that were $\mathrm{CK}^{-} / \mathrm{CD} 45^{-} / \mathrm{DAPI}^{+} / \mathrm{CEP} 8=2$ spots were recognized as 

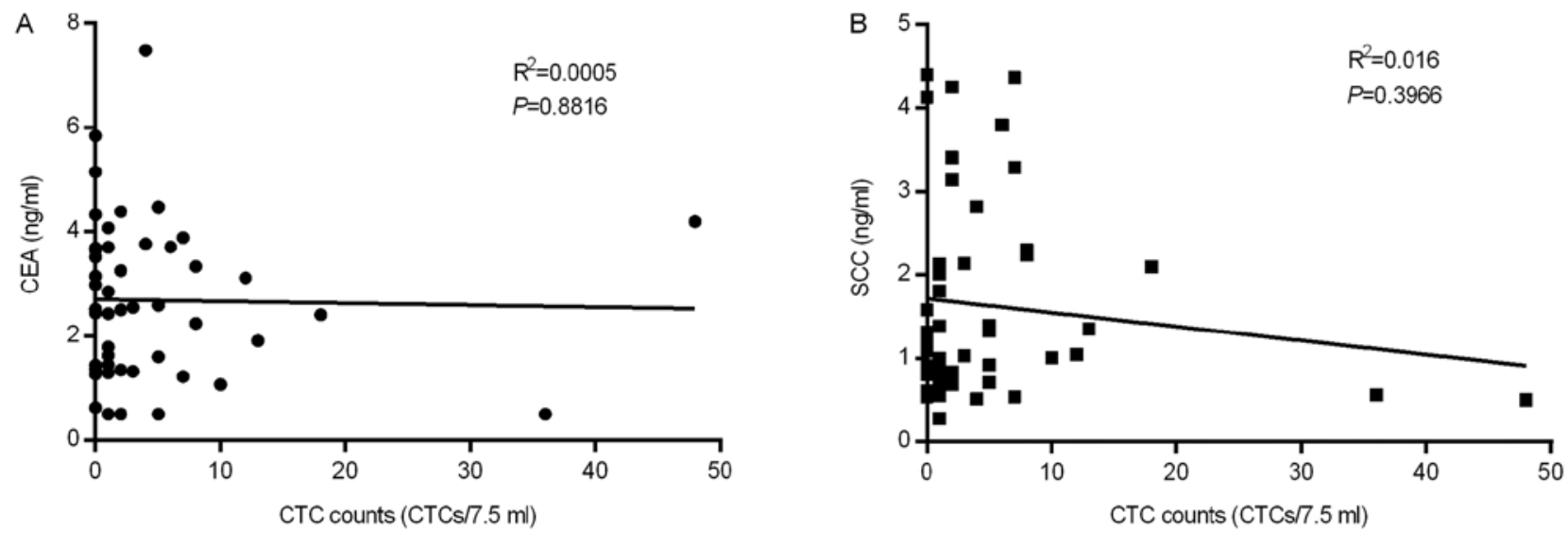

Figure 3. Comparison of CTCs and serological tumor markers as blood-based markers. (A) Spearman correlation between CTC counts and CEA concentration. (B) Spearman correlation between CTC counts and SCC antigen concentration. CTCs, circulating tumor cells; CEA, carcinoembryonic antigen; SCC, squamous cell carcinoma.

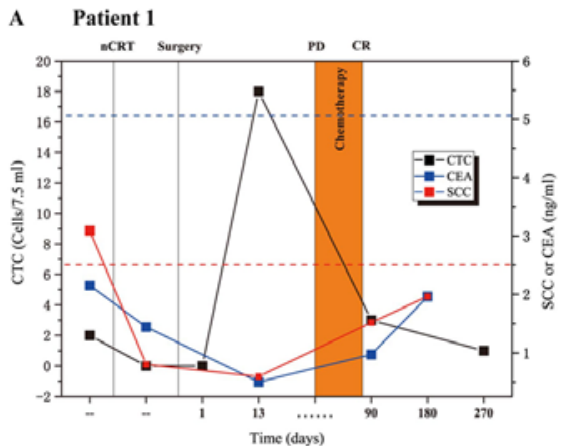

D

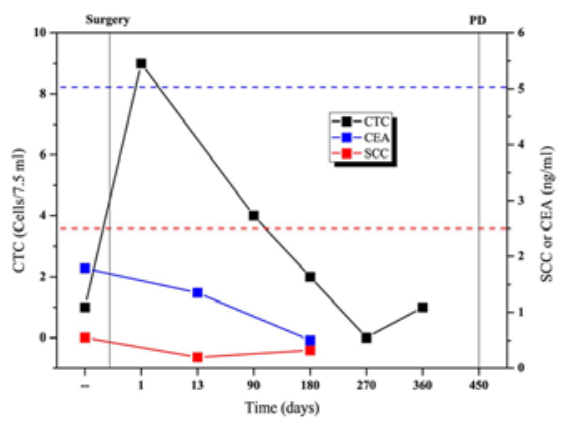

B Patient 3

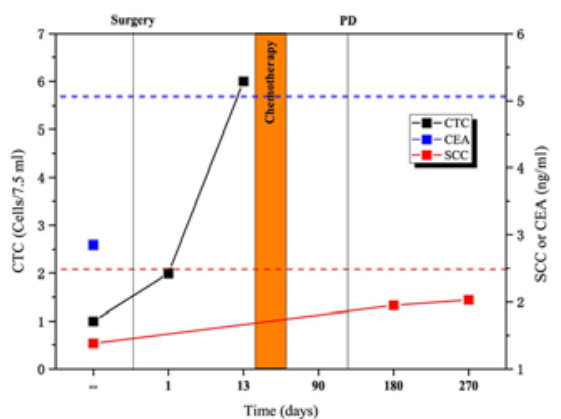

E Patient 18

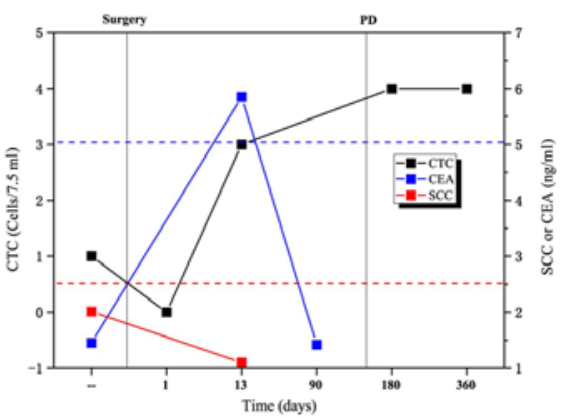

C Patient 11

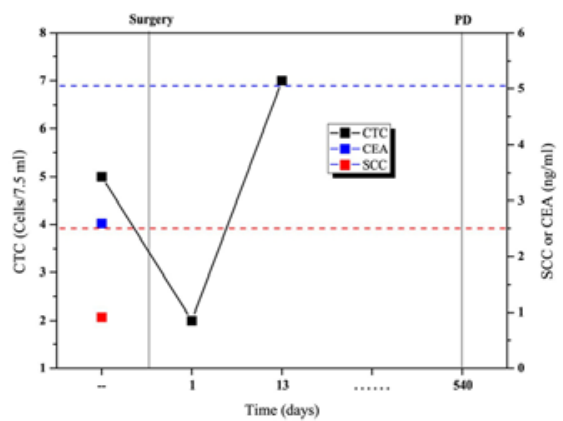

F Patient 44

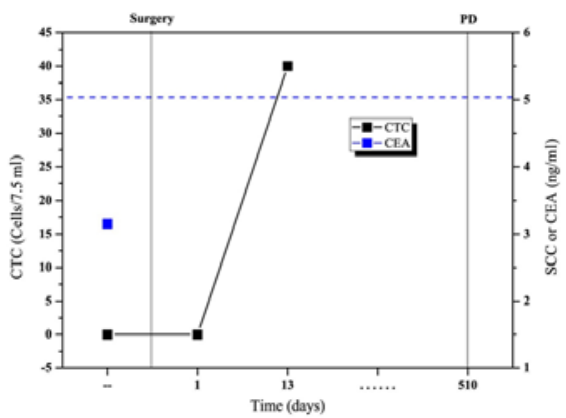

Figure 4. Dynamic monitoring of CTC counts and serological tumor markers in six patients with esophageal cancer pre- and post-operative care. The six patients were selected since they periodically underwent CTC detection and were identified as having relapsed ESCC during the follow-up period. Patient (A) 1 , (B) 3, (C) 11, (D) 12, (E) 18 and (F) 44 were selected. The dotted blue line indicates CEA cut-off level (5.09 ng/ml); the dotted red line indicates SCC antigen cut-off level (2.5 ng/ml). CEA, carcinoembryonic antigen; CR, complete response; CTCs, circulating tumor cells; NCRT, neoadjuvant chemoradiotherapy; PD, progressive disease; SCC, squamous cell carcinoma.

indeterminate cells, which could be either $\mathrm{CK}^{-}$tumor cells or WBCs with unstained CD45. In the present study, $\mathrm{CK}^{+} \mathrm{CTCs}$ accounted for a very small proportion of identified CTCs; this has also been reported by previous studies $(28,29,40)$. Due to its higher detection rate, iFISH may be more clinically useful than the CK staining method using anti-CK8/18/19 and anti-CD45 antibodies used in other studies $(19,20)$.

CTCs are derived from primary or metastatic lesions, and metastasis begins with epithelial-mesenchymal transition (EMT). Chen et al (41) and Han et al (42) demonstrated the importance of the EMT process in tumor metastasis and CTC production; however, CTC clusters, also known as microemboli, were not detected in any of the cohorts enrolled in these studies $(41,42)$. Clustered cancer cells in the blood have been reported to serve as an indicator of poor prognosis and early recurrence in lung cancer $(43,44)$, and high malignancy in breast cancer $(30,45)$; however, to the best of our knowledge, they have not been studied in ESCC. In the present study, two CTC clusters in two patients were identified separately prior to surgery, and another three clustered CTCs were detected in three patients following resection (data not shown). Among these patients, CT detected recurrence in only one patient from whom clustered CTCs were detected prior to pre-operative chemoradiotherapy. No CTC clusters were detected in the 
Table II. Univariate and multivariate Cox proportional hazards regression analyses for prediction of progression-free survival.

\begin{tabular}{|c|c|c|c|c|c|c|}
\hline \multirow[b]{2}{*}{ Risk factor } & \multicolumn{3}{|c|}{ Univariate analysis } & \multicolumn{3}{|c|}{ Multivariate analysis } \\
\hline & P-value & HR & $95 \% \mathrm{CI}$ & P-value & HR & $95 \%$ CI \\
\hline$\Delta \mathrm{CTC} \geq 2$ & 0.073 & 3.184 & $0.898-11.294$ & 0.047 & 3.922 & $0.907-16.951$ \\
\hline Advanced stage & 0.072 & 2.583 & $0.919-7.265$ & 0.101 & 12.001 & $0.613-234.850$ \\
\hline LNM & 0.093 & 2.387 & $0.865-6.588$ & 0.206 & 0.128 & $0.005-3.101$ \\
\hline Smoking & 0.102 & 2.559 & $0.815-8.039$ & 0.082 & 7.447 & $0.775-71.595$ \\
\hline Drinking & 0.042 & 2.924 & $1.040-8.217$ & 0.951 & 1.048 & $0.240-4.574$ \\
\hline
\end{tabular}

$\triangle \mathrm{CTC}=\mathrm{CTC}_{13}-\mathrm{CTC}_{\mathrm{FD}}$; where $\mathrm{CTC}_{13}$ refers to CTC counts 13 days post-surgery, and $\mathrm{CTC}_{\mathrm{FD}}$ refers to CTC counts at first diagnosis. $\mathrm{CI}$, confidence interval; CTC, circulating tumor cell; HR, hazard ratio; LNM, lymph node metastasis.

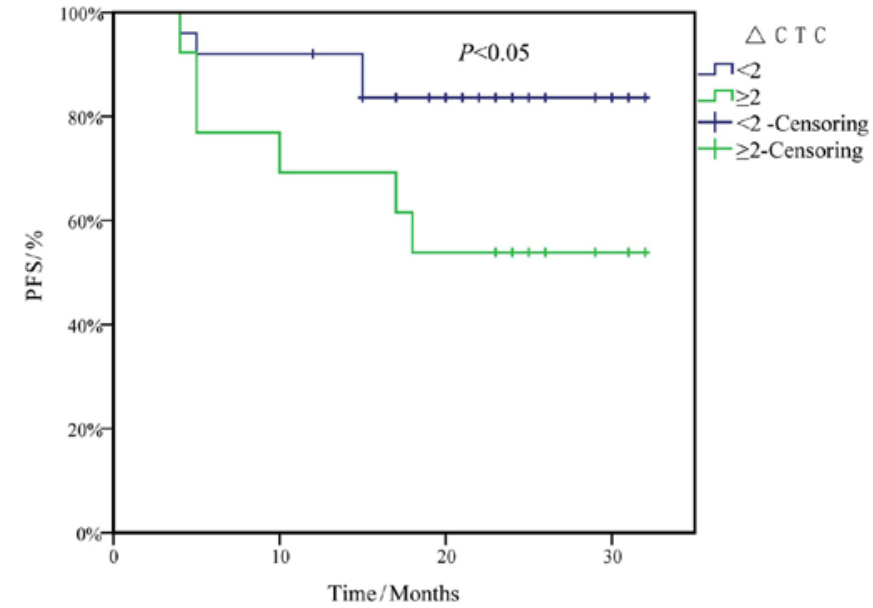

Figure 5. Kaplan-Meier curve analysis of PFS in patients with different cut-off values of CTCs. The cut-off value was defined as $\Delta \mathrm{CTC}=2 / 7.5 \mathrm{ml}$ $\left(\Delta \mathrm{CTC}=\mathrm{CTC}_{13}-\mathrm{CTC}_{\mathrm{FD}}\right)$. CTCs, circulating tumor cells; $\mathrm{CTC}_{13}, \mathrm{CTC}$ counts 13 days post-surgery; $\mathrm{CTC}_{\mathrm{FD}}$, $\mathrm{CTC}$ counts at first diagnosis; PFS, progression-free survival.

enrolled patients 3 months post-surgery. A total of two clustered CTCs were detected in patients $24 \mathrm{~h}$ following resection, which suggested that surgery may dislodge cells, resulting in their movement from the resection site to the circulation, thus inducing the formation of clustered CTCs. Inflammatory conditions generated by resection may also contribute to the increased clusters (46); this may also explain why no clusters were detected in any of the patients 3 months post-surgery when inflammation was reduced. These clusters could be more apoptosis-resistant and metastatic once they co-express epithelial and mesenchymal markers (30). A more in-depth approach would be to characterize these EMT markers during the entire study, since it may help to explain why the three patients with clusters following surgery had no recurrence, whereas one patient with clustered CTCs prior to treatment exhibited recurrence. The significance of the present findings is limited by the lack of EMT marker assessment and the small number of patients with detected clusters; therefore, no significant association between CTC clusters and prognosis or PFS was identified. Nevertheless, further research is required in order to confirm that pre-operative, instead of post-operative CTC clusters, are associated with ESCC recurrence, as such insight may facilitate the full understanding of clustered CTCs and ESCC prognosis.

Another clinical problem the present study aimed to solve was the monitoring time points. To understand at which stage CTCs are associated with treatment response, the detection of CTCs or other serum protein markers was performed at multiple time points, during the pre-operative and post-operative period. Compared with SCC antigen, CEA or CT imaging, CTC fluctuation in the patients reflected earlier recurrence of esophageal cancer. CTC counts increased in $>50 \%$ of patients $24 \mathrm{~h}$ post-surgery, which further verified the hypothesis that the dislodged primary tumor cells from surgery may enter the circulation and interfere with CTC enumeration. In this case, the results of CTC detection cannot be applied for evaluating therapeutic effect or prognosis. Compared to the other time points, CTC counts 13 days post-resection were a more favorable indicator, since the dislodged tumor cells have probably undergone apoptosis during this period; therefore, only the resistant cells may survive and induce metastasis, which is responsible for potential disease progression.

Taking this into consideration, Kaplan-Meier and Cox analyses were performed to analyze the predictive effects of CTC counts on PFS. Instead of considering a certain CTC number prior to surgery the cut-off value to predict prognosis, multivariate analysis revealed that $\triangle C T C$ counts $\geq 2 / 7.5 \mathrm{ml}$ PB may be a strong prognostic indicator of PFS (HR, 3.922; 95\% CI, 0.907-16.951; $\mathrm{P}<0.05)$ in patients with ESCC. Patients with a change in CTC status from negative (0 CTC at first diagnosis) to negative (0 CTC on day 13 post-surgery) had the best prognosis [0\% progressive disease (PD)], whereas patients with positive CTCs at pre- and post-operative times points had the worst prognosis (36\% PD, data not shown). Even patients with a variation in CTC counts from positive to negative underwent metastasis or recurrence, which was contradictory to the results of previous studies $(19,20)$. This finding is of great clinical significance, as the cut-off value for predicting prognosis was established with variation of CTC counts $\left(\triangle \mathrm{CTC}=\mathrm{CTC}_{13}-\mathrm{CTC}_{\mathrm{FD}}\right)$, instead of CTC number at a certain time point.

In conclusion, the findings of the present study are limited due to the small number of patients and the short follow-up period. A prospective study with a larger sample size and a longer follow-up period is necessary, in order to evaluate the 
clinical significance of the SE-iFISH system in CTC detection for patients with ESCC. In addition, the clusters of CTCs need to be isolated and characterized for different cell types present, and the results subsequently compared with patient prognosis. In addition, the application of next-generation sequencing and single-cell sequencing CTCs to monitor genomic variations in disease progression, and decipher tumor evolution during treatment, as well as the EMT mechanism, has been reported in several types of cancer (47-49). These aforementioned findings provide potential direction for future studies. With further technical improvements in detection and sequencing methods, CTC analyses may exhibit greater potential as biomarkers for evaluating cancer prognosis and treatment response.

\section{Acknowledgements}

Not applicable.

\section{Funding}

This study was supported by the Key Project of National Natural Science Foundation of China (grant no. U1504814), Major Projects of Science and Technology Department in Henan Province (grant nos. 16110311200 and 161100311300), the Project of Anyang Science Foundation of Henan province (grant no. 2016) and the Major Projects of Special Development Funds in Zhangjiang National Independent Innovation Demonstration Zone, Shanghai (grant no. ZJ2017-ZD-012).

\section{Availability of data and materials}

The datasets used and/or analyzed during the current study available from the corresponding author on reasonable request.

\section{Authors' contributions}

FZ and RF developed the study concepts and design, and were responsible for quality control of data and algorithms. PH, JX, JZ, JX, LDW, FS and AZ participated in data acquisition. YZ, LW and JZ conducted the statistical analysis. YZ, PM and JL were responsible for interpreting data and preparation of the manuscript, including drafting and revising the manuscript. All authors edited and reviewed the final manuscript. All authors read and approved the final manuscript and agree to be accountable for all aspects of the work.

\section{Ethics approval and consent to participate}

This study was approved by the Ethics Committee of Anyang Cancer Hospital, and written informed consent was obtained from all participants.

\section{Patient consent for publication}

Not applicable.

\section{Competing interests}

The authors declare that they have no competing interests.

\section{References}

1. Bohanes P, Yang D, Chhibar RS, Labonte MJ, Winder T, Ning Y, Gerger A, Benhaim L, Paez D, Wakatsuki T, et al: Influence of sex on the survival of patients with esophageal cancer. J Clin Oncol 30: 2265-2272, 2012.

2. Torre LA, Bray F, Siegel RL, Ferlay J, Lortet-Tieulent J and Jemal A: Global cancer statistics, 2012. CA Cancer J Clin 65: 87-108, 2015.

3. Abnet CC, Arnold M and Wei WQ: Epidemiology of esophageal squamous cell carcinoma. Gastroenterology 154: 360-373, 2018.

4. Matsushima K, Isomoto H, Yamaguchi N, Inoue N, Machida H, Nakayama T, Hayashi T, Kunizaki M, Hidaka S, Nagayasu T, et al: MiRNA-205 modulates cellular invasion and migration via regulating zinc finger E-box binding homeobox 2 expression in esophageal squamous cell carcinoma cells. J Transl Med 9: 30, 2011.

5. Nagaraja V and Eslick GD: Forthcoming prognostic markers for esophageal cancer: A systematic review and meta-analysis. J Gastrointest Oncol 5: 67-76, 2014.

6. Pera M, Manterola C, Vidal O and Grande L: Epidemiology of esophageal adenocarcinoma. J Surg Oncol 92: 151-159, 2005.

7. Fan YJ, Song X, Li JL, Li XM, Liu B, Wang R, Fan ZM and Wang LD: Esophageal and gastric cardia cancers on 4238 Chinese patients residing in municipal and rural regions: a histopathological comparison during 24-year period. World J Surg 32: 1980-1988, 2008.

8. Pennathur A, Gibson MK, Jobe BA and Luketich JD: Oesophageal carcinoma. Lancet 381: 400-412, 2013.

9. Enzinger PC and Mayer RJ: Esophageal cancer. N Engl J Med 349: 2241-2252, 2003.

10. Domper Arnal MJ, Ferrandez Arenas A and Lanas Arbeloa A: Esophageal cancer: Risk factors, screening and endoscopic treatment in Western and Eastern countries. World J Gastroenterol 21: 7933-7943, 2015

11. Zhang J, Zhu Z, Liu Y, Jin X, Xu Z, Yu Q and Li K: Diagnostic value of multiple tumor markers for patients with esophageal carcinoma. PLoS One 10: e0116951, 2015.

12. He Z, Liu Z, Liu M, Guo C, Xu R, Li F, Liu A, Yang H, Shen L, $\mathrm{Wu} \mathrm{Q}$, et al: Efficacy of endoscopic screening for esophageal cancer in China (ESECC): Design and preliminary results of a population-based randomised controlled trial. Gut 68: 198-206, 2019.

13. Li J, Xu R, Liu M, Cai H, Cao C, Liu F, Li F, Guo C, Pan Y, He Z and Ke Y: Lugol chromoendoscopy detects esophageal dysplasia with low levels of sensitivity in a high-risk region of china. Clin Gastroenterol Hepatol 16: 1585-1592, 2018.

14. Smyth EC, Lagergren J, Fitzgerald RC, Lordick F, Shah MA, Lagergren $P$ and Cunningham D: Oesophageal cancer. Nat Rev Dis Primers 3: 17048, 2017.

15. Zhang Y, Wang F, Ning N, Chen Q, Yang Z, Guo Y, Xu D, Zhang D, Zhan T and Cui W: Patterns of circulating tumor cells identified by CEP8, CK and CD45 in pancreatic cancer. Int J Cancer 136: 1228-1233, 2015.

16. Marx V: Tracking metastasis and tricking cancer. Nature 494: 133-136, 2013.

17. Kim MY, Oskarsson T, Acharyya S, Nguyen DX, Zhang XH, Norton $\mathrm{L}$ and Massagué J: Tumor self-seeding by circulating cancer cells. Cell 139: 1315-1326, 2009.

18. Pierga JY,Bidard FC, Mathiot C, Brain E, Delaloge S, Giachetti S, de Cremoux P, Salmon R, Vincent-Salomon A and Marty M: Circulating tumor cell detection predicts early metastatic relapse after neoadjuvant chemotherapy in large operable and locally advanced breast cancer in a phase II randomized trial. Clin Cancer Res 14: 7004-7010, 2008.

19. Matsushita D, Uenosono Y, Arigami T, Yanagita S, Nishizono Y, Hagihara T, Hirata M, Haraguchi N, Arima H, Kijima Y, et al: Clinical significance of circulating tumor cells in peripheral blood of patients with esophageal squamous cell carcinoma. Ann Surg Oncol 22: 3674-3680, 2015.

20. Qiao Y, Li J, Shi C, Wang W, Qu X, Xiong M, Sun Y, Li D, Zhao X and Zhang D: Prognostic value of circulating tumor cells in the peripheral blood of patients with esophageal squamous cell carcinoma. Onco Targets Ther 10: 1363-1373, 2017.

21. Lianidou ES and Markou A: Circulating tumor cells as emerging tumor biomarkers in breast cancer. Clin Chem Lab Med 49: 1579-1590, 2011.

22. Swennenhuis JF, van Dalum G, Zeune LL and Terstappen LW: Improving the CellSearch ${ }^{(\mathrm{R})}$ system. Expert Rev Mol Diagn 16: 1291-1305, 2016. 
23. Andree KC, van Dalum G and Terstappen LW: Challenges in circulating tumor cell detection by the CellSearch system. Mol Oncol 10: 395-407, 2016

24. Sheng Y, Wang T, Li H, Zhang Z, Chen J, He C, Li Y, Lv Y, Zhang J, Xu C, et al: Comparison of analytic performances of Cellsearch and iFISH approach in detecting circulating tumor cells. Oncotarget 8: 8801-8806, 2017.

25. Reeh M, Effenberger KE, Koenig AM, Riethdorf S, Eichstädt D, Vettorazzi E, Uzunoglu FG, Vashist YK, Izbicki JR, Pantel K and Bockhorn M: Circulating tumor cells as a biomarker for preoperative prognostic staging in patients with esophageal cancer. Ann Surg 261: 1124-1130, 2015.

26. Stephen Edge DRB and Carolyn C: AJCC Cancer Staging Manual (7th edition). Edge S, Byrd DR, Compton CC, Fritz AG, Greene F and Trotti A (eds). Springer, New York, NY, 2010.

27. Liu X, Zhang Z, Zhang B, Zheng Y, Zheng C, Liu B, Zheng S, Dong K and Dong R: Circulating tumor cells detection in neuroblastoma patients by EpCAM-independent enrichment and immunostaining-fluorescence in situ hybridization. EBioMedicine 35: 244-250, 2018.

28. Wu W, Zhang Z, Gao XH, Shen Z, Jing Y, Lu H, Li H, Yang X, Cui X, Li Y, et al: Clinical significance of detecting circulating tumor cells in colorectal cancer using subtraction enrichment and immunostaining-fluorescence in situ hybridization (SE-iFISH). Oncotarget 8: 21639-21649, 2017.

29. Xu L, Jia S, Li H, Yu Y, Liu G, Wu Y, Liu X, Liu C, Zhou Y, Zhang Z and Sheng Y: Characterization of circulating tumor cells in newly diagnosed breast cancer. Oncol Lett 15: 2522-2528, 2018.

30. Aceto N, Bardia A, Miyamoto DT, Donaldson MC, Wittner BS, Spencer JA, Yu M, Pely A, Engstrom A, Zhu H, et al: Circulating tumor cell clusters are oligoclonal precursors of breast cancer metastasis. Cell 158: 1110-1122, 2014.

31. Therasse P, Arbuck SG, Eisenhauer EA, Wanders J, Kaplan RS, Rubinstein L, Verweij J, Van Glabbeke M, van Oosterom AT, Christian MC and Gwyther SG: New guidelines to evaluate the response to treatment in solid tumors. European Organization for Research and Treatment of Cancer, National Cancer Institute of the United States, National Cancer Institute of Canada. J Natl Cancer Inst 92: 205-216, 2000.

32. Nagrath S, Sequist LV, Maheswaran S, Bell DW, Irimia D, Ulkus L, Smith MR, Kwak EL, Digumarthy S, Muzikansky A, et al: Isolation of rare circulating tumour cells in cancer patients by microchip technology. Nature 450: 1235-1239, 2007.

33. Masuda T, Hayashi N, Iguchi T, Ito S, Eguchi $\mathrm{H}$ and Mimori K: Clinical and biological significance of circulating tumor cells in cancer. Mol Oncol 10: 408-417, 2016.

34. Yap TA, Lorente D, Omlin A, Olmos D and de Bono JS: Circulating tumor cells: A multifunctional biomarker. Clin Cancer Res 20: 2553-2568, 2014.

35. Cristofanilli M, Budd GT, Ellis MJ, Stopeck A, Matera J, Miller MC, Reuben JM, Doyle GV, Allard WJ, Terstappen LW and Hayes DF: Circulating tumor cells, disease progression and survival in metastatic breast cancer. N Engl J Med 351: 781-791, 2004.

36. Vona G, Sabile A, Louha M, Sitruk V, Romana S, Schütze K, Capron F, Franco D, Pazzagli M, Vekemans M, et al: Isolation by size of epithelial tumor cells: A new method for the immunomorphological and molecular characterization of circulatingtumor cells. Am J Pathol 156: 57-63, 2000
37. Xue F, Shi S, Zhang Z, Xu C, Zheng J, Qin T, Qian Z, Zhao X, Tong Y, Xia L and Xia Q: Application of a novel liquid biopsy in patients with hepatocellular carcinoma undergoing liver transplantation. Oncol Lett 15: 5481-5488, 2018.

38. Roach T, Slater S, Koval M, White L, Cahir McFarland ED, Okumura M, Thomas M and Brown E: CD45 regulates Src family member kinase activity associated with macrophage integrin-mediated adhesion. Curr Biol 7: 408-417, 1997.

39. Court CM, Ankeny JS, Hou S, Tseng HR and Tomlinson JS: Improving pancreatic cancer diagnosis using circulating tumor cells: Prospects for staging and single-cell analysis. Expert Rev Mol Diagn 15: 1491-1504, 2015.

40. Ge F, Zhang H, Wang DD, Li L and Lin PP: Enhanced detection and comprehensive in situ phenotypic characterization of circulating and disseminated heteroploid epithelial and glioma tumor cells. Oncotarget 6: 27049-27064, 2015.

41. Chen W, Li Y, Yuan D, Peng Y and Qin J: Practical value of identifying circulating tumor cells to evaluate esophageal squamous cell carcinoma staging and treatment efficacy. Thorac Cancer 9: 956-966, 2018

42. Han D, Chen K, Che J, Hang J and Li H: Detection of epithelial-mesenchymal transition status of circulating tumor cells in patients with esophageal squamous carcinoma. Biomed Res Int 2018: 7610154, 2018.

43. Wang J, Wang K, Xu J, Huang J and Zhang T: Prognostic significance of circulating tumor cells in non-small-cell lung cancer patients: A meta-analysis. PLoS One 8: e78070, 2013.

44. Funaki S, Sawabata N, Abulaiti A, Nakagiri T, Shintani Y, Inoue M, Minami M and Okumura M: Significance of tumour vessel invasion in determining the morphology of isolated tumour cells in the pulmonary vein in non-small-cell lung cancer. Eur J Cardiothorac Surg 43: 1126-1130, 2013.

45. Yu M, Bardia A, Wittner BS, Stott SL, Smas ME, Ting DT, Isakoff SJ, Ciciliano JC, Wells MN, Shah AM, et al: Circulating breast tumor cells exhibit dynamic changes in epithelial and mesenchymal composition. Science 339: 580-584, 2013.

46. Jolly MK, Boareto M, Huang B, Jia D, Lu M, Ben-Jacob E, Onuchic JN and Levine $\mathrm{H}$ : Implications of the Hybrid epithelial/mesenchymal phenotype in metastasis. Front Oncol 5: $155,2015$.

47. Gao Y, Ni X, Guo H, Su Z, Ba Y, Tong Z, Guo Z, Yao X, Chen X, Yin J, et al: Single-cell sequencing deciphers a convergent evolution of copy number alterations from primary to circulating tumor cells. Genome Res 27: 1312-1322, 2017.

48. D'Avola D, Villacorta-Martin C, Martins-Filho SN, Craig A, Labgaa I, von Felden J, Kimaada A, Bonaccorso A, Tabrizian P, Hartmann BM, et al: High-density single cell mRNA sequencing to characterize circulating tumor cells in hepatocellular carcinoma. Sci Rep 8: 11570, 2018.

49. Zhu Z, Qiu S, Shao K and Hou Y: Progress and challenges of sequencing and analyzing circulating tumor cells. Cell Biol Toxicol 34: 405-415, 2017.

This work is licensed under a Creative Commons Attribution-NonCommercial-NoDerivatives 4.0 International (CC BY-NC-ND 4.0) License. 